\title{
The Fifth Workshop on Galactic Chemodynamics
}

\author{
Brad K. Gibson ${ }^{1,2}$ and Daisuke Kawata ${ }^{1}$ \\ ${ }^{1}$ Centre for Astrophysics \& Supercomputing, Swinburne University, Hawthorn VIC 3122, Australia \\ ${ }^{2}$ E-mail: bgibson@astro.swin.edu.au
}

From 1992 to 1995, the annual Workshops on Galactic Chemodynamics were recognised as the primary crossdisciplinary meetings for theorists and observers interested in understanding the formation and evolution of galaxies — in particular, the Milky Way. ${ }^{1}$ The eight years subsequent to the Fourth Workshop have seen an extraordinary expansion in the field of chemodynamics, driven not only by the obvious advances in computational power, but by an incredible wealth of new observational data ranging from the discovery of multiple chemo-kinematical substructures in the Milky Way and M31 halos, to the discovery of the most metal-poor object known in the Universe (also within the halo of the Milky Way). Further, the recognition that "near-field cosmology" — deconstructing the formation and evolution history of our Milky Way on a star-by-star basis — was the primary science driver for ambitious next-generation surveys and facilities such as the RAdial Velocity Experiment $\left(\right.$ RAVE) ${ }^{2}$ and the European Space Agency's Gaia mission ${ }^{3}$, led us to believe that a Fifth Workshop on Galactic Chemodynamics (GCDV) was long overdue.

In September 2002, we put out a call for participation in GCDV and were overwhelmed with the number of positive responses. Our goal with GCDV was to once again bring together the leaders in the computational and observational fields, in order to galvanise efforts related to deconstructing the history of formation of the Milky Way.

GCDV took place during 9-11 July 2003, at Swinburne University, Melbourne, Australia. A remarkable 46 participants from 13 countries attended the Workshop; 28 of the 36 talks presented there have been included in these proceedings.

The broad topics covered during the meeting included:

- the formation of the Milky Way in a Cold Dark Matter universe: merging versus smooth accretion

- the connection between the halo, bulge, and thick + thin disk components

- correlations between chemical and dynamical properties of stars in the Milky Way

\footnotetext{
${ }^{1}$ The locations for the first four Workshops on Galactic Chemodynamics were Clemson (1992), Kiel (1993), Livermore (1994), and Ringberg (1995).

2 http: / / astronomy. swin. edu.au/RAVE/

${ }^{3}$ http: //astro.estec.esa.nl/GAIA/
}

Table 1. List of participants, home institutions, and manuscripts

\begin{tabular}{|c|c|c|}
\hline Johannes Andersen & Copenhagen & p. 129 \\
\hline Dominik Argast & Basel & p. 161 \\
\hline Tim Beers & Michigan State & p. 207 \\
\hline Kenji Bekki & UNSW & p. 167 \\
\hline Joss Bland-Hawthorn & $\mathrm{AAO}$ & p. 110 \\
\hline Masashi Chiba & Tohoku & p. 237 \\
\hline Chris Brook & Swinburne & p. 153 \\
\hline David Burstein & Arizona State & \\
\hline Bruce Carney & North Carolina & p. 134 \\
\hline Tim Connors & Swinburne & p. 222 \\
\hline Lisa Elliott & Monash & \\
\hline Chris Flynn & Tuorla & pp 126,153 \\
\hline Ken Freeman & ANU & p. 110 \\
\hline Ortwin Gerhard & Basel & \\
\hline Brad Gibson & Swinburne & pp $153,216,222$ \\
\hline Stefan Harfst & Kiel & p. 228 \\
\hline Amina Helmi & Groningen & p. 212 \\
\hline Gerhard Hensler & Vienna & pp 188,228 \\
\hline Janne Holopainen & Tuorla & p. 153 \\
\hline Akihiko Ibukiyama & NAOJ & p. 121 \\
\hline Inese Ivans & Caltech & \\
\hline Daisuke Kawata & Swinburne & pp 153,222 \\
\hline Alexander Knebe & Swinburne & p. 216 \\
\hline Chiaki Kobayashi & MPA & p. 183 \\
\hline Hyun-chul Lee & Swinburne & p. 153 \\
\hline Geraint Lewis & Sydney & p. 203 \\
\hline Steve Majewski & Virginia & p. 197 \\
\hline Masao Mori & Senshu & p. 232 \\
\hline Naohito Nakasato & Tokyo & p. 171 \\
\hline Julio Navarro & Victoria & \\
\hline Birgitta Nordström & Lund & p. 129 \\
\hline Laura Portinari & TAC & p. 144 \\
\hline Simone Recchi & MPA & p. 157 \\
\hline Agostino Renda & Swinburne & p. 153 \\
\hline Céline Reylé & Besançon & p. 138 \\
\hline Markus Samland & Basel & pp 161,175 \\
\hline Arnaud Siebert & Arizona & \\
\hline Rainer Spurzem & Heidelberg & p. 188 \\
\hline Takeru Suzuki & Kyoto & p. 148 \\
\hline Matthias Steinmetz & Potsdam & \\
\hline Christian Theis & Vienna & pp $179,188,228$ \\
\hline Chris Thom & Swinburne & \\
\hline Patricia Tissera & IAFE & p. 192 \\
\hline Eline Tolstoy & Groningen & \\
\hline Takuji Tsujimoto & NAOJ & p. 242 \\
\hline Hideki Yahagi & NAOJ & \\
\hline
\end{tabular}


- the (homogeneous and/or inhomogeneous) chemical enrichment history of galaxies

- evolution of the Galactic multi-phase interstellar medium

- self-regulating star formation and chemodynamics

- computational methods of galaxy evolution ( $N$-body, Smoothed Particle Hydrodynamics, Adaptive Mesh Refinement).

The Scientific Organising Committee was charged with ensuring that the ambitious scientific scope was met, and as the 28 papers presented in these proceedings demonstrate, they appear to have succeeded. The SOC was Chaired by Brad Gibson (Swinburne), and supported ably by Andi Burkert (Munich), Gerhard Hensler (Vienna), and Daisuke Kawata (Swinburne).

The success of GCDV can be traced in no small part to the support provided by the Local Organising Committee. While Chaired by Brad Gibson and Daisuke Kawata, it was really the efforts of Michelle Jolley, Chris Brook, and Chris Thom that allowed the Workshop to function seamlessly.

The publication of the proceedings of GCDV was made possible through the support of the Publications of the
Astronomical Society of Australia. We especially wish to acknowledge the tireless efforts of its editors Louise Hartley and Richard Hecker. Further, while Brad Gibson and Daisuke Kawata oversaw the editorial duties for these particular proceedings, their editorial assistants - Chris Brook, Yeshe Fenner, Agostino Renda, and Chris Thom deserve special mention for chasing down authors and referee reports for each of the manuscripts.

Finally, we would like to express our thanks to each of the Workshop participants. The overwhelming response to the Call for a Fifth Workshop on Galactic Chemodynamics (we had anticipated fewer than 20 interested participants!) demonstrates the need for the community to gather for such targeted meetings on a more regular basis. We can only hope that the primary legacy of GCDV will be the re-establishment of an annual (or at least biennial) Galactic Chemodynamics Workshop series - it is clear that the demand and desire exists.
Brad K. Gibson

Daisuke Kawata June 2004 